\title{
ON THE INITIAL VALUE PROBLEM FOR FRACTIONAL VOLTERRA INTEGRODIFFERENTIAL EQUATIONS WITH A CAPUTO-FABRIZIO DERIVATIVE
}

\author{
Nguyen Huy Tuan ${ }^{1}$, Nguyen Anh Tuan ${ }^{2}$, Donal O'Regan ${ }^{3}$ \\ AND VO VIET TRI ${ }^{2, *}$
}

\begin{abstract}
In this paper, a time-fractional integrodifferential equation with the Caputo-Fabrizio type derivative will be considered. The Banach fixed point theorem is the main tool used to extend the results of a recent paper of Tuan and Zhou [J. Comput. Appl. Math. 375 (2020) 112811]. In the case of a globally Lipschitz source terms, thanks to the $L^{p}-L^{q}$ estimate method, we establish global in time well-posed results for mild solution. For the case of locally Lipschitz terms, we present existence and uniqueness results. Also, we show that our solution will blow up at a finite time. Finally, we present some numerical examples to illustrate the regularity and continuation of the solution based on the time variable.
\end{abstract}

Mathematics Subject Classification. 26A33, 35B65, 35R11.

Received June 30, 2020. Accepted January 24, 2021.

\section{INTRODUCTION}

Fractional calculus has many applications in mechanic, physics and engineering science... For example, a fractional diffusion equation is a generalization of a classical diffusion equation which models anomalous diffusive phenomena. For results on fractional derivatives like Riemann-Liouville type or Caputo type we refer the reader to $[1,13,23,29,31,35]$ and the references therein. Recently Caputo and Fabrizio [16] introduced a new fractional derivative, which called Caputo-Fabrizio fractional derivative.

Our paper focuses on studying the existence and uniqueness of a mild solution of time-fractional Volterra integrodifferential equations with the new type of fractional derivative. Let $T$ be a positive number and $\alpha \in(0,1)$, we consider the following intial value problem

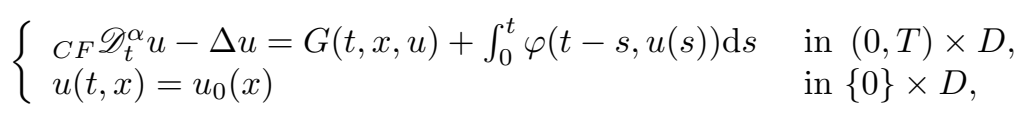

Keywords and phrases: Fractional nonclassical diffusion equation, well-posednes, regularity estimates.

1 Applied Analysis Research Group, Faculty of Mathematics and Statistics, Ton Duc Thang University, Ho Chi Minh City, Vietnam.

2 Division of Applied Mathematics, Thu Dau Mot University, Binh Duong Province, Vietnam.

3 School of Mathematics, Statistics and Applied Mathematics, National University of Ireland, Galway, Ireland.

* Corresponding author: trivv@tdmu.edu.vn 
here, $u_{0}$ is the initial data, the functions $G, \varphi$ and the domain $D$ will be defined later. The symbol ${ }_{C F} \mathscr{D}_{t}^{\alpha}$ stands for the Caputo-Fabrizio type time fractional derivative operator of order $\alpha$ (see [15, 39]). We recall the definition of the Caputo-Fabrizio type time fractional derivative as follows. Let $a>0,0 \leq \alpha \leq 1$, and for a function $w$ belongs to $H^{1}(0, a)$, its Caputo-Fabrizio fractional derivative is defined as (see [15])

$$
{ }_{C F} \mathscr{D}_{t}^{\alpha} w(t)=\frac{M(\alpha)}{1-\alpha} \int_{0}^{t} \exp \left(-\frac{\alpha s}{1-\alpha}\right) \frac{\partial w}{\partial s} \mathrm{~d} s, \quad t \geq 0
$$

where $M$ is a normalization function such that $M(0)=M(1)=1$. When $\varphi=0$, the authors in [39] investigated the well-posedness of an initial value problem for a fractional diffusion equation. In [2], the existence of the solution to an initial value problem for a linear differential equation was investigated and the authors applied their results to the mass-spring-damper motion in the general case. We refer the reader to [25] for results on the existence of the Korteweg-de Vries-Burgers equation with a fractional Caputo-Fabrizio derivative. For more results on time fractional partial derivative equations topic, we refer the reader to $[5-7,9-12,26,27,32,36,38]$.

When $(\varphi \neq 0)$, problem $(1.1)$ can be used to model some natural phenomena with memory effects. In [3] the authors considered memory effects on the dynamics of non-Newtonian fluids and viscoelastic models for the dynamics of turbulence statistics in Newtonian fluids on the modified 3D Navier-Stokes equation

$$
\begin{array}{ll}
u_{t}=\Delta u-\nabla p-(u \cdot \nabla) u+f(t)+\int_{0}^{t} a(t-s)(-\Delta)^{\beta} u(s) \mathrm{d} s, & x \in \Omega \\
\operatorname{div}(u)=0, & x \in \Omega, \\
u=0, & x \in \partial \Omega .
\end{array}
$$

In [34] the authors compared the energy dissipation produced by the internal motion and the memory effect i.e. they studied the behavior between the equation

$$
u_{t t}+\Delta^{2} u+\alpha \Delta u_{t}=0 \quad \text { in } \Omega
$$

and the equation

$$
u_{t t}+\Delta^{2} u-\int_{0}^{t} g(t-\tau) \Delta^{2} u(\cdot, \tau) \mathrm{d} \tau=0 \quad \text { in } \Omega .
$$

We also refer the reader to $[20,28,30,40]$ and the references therein. In [20] the authors established the existence of global and exponential attractors of optimal regularity and finite fractal dimension for the related semigroup of solutions of the equation

$$
\partial_{t} u-\Delta u-\int_{0}^{\infty} \kappa(s) \Delta u(t-s) \mathrm{d} s+\varphi(u)=f,
$$

which arises in the Coleman-Gurtin theory of heat conduction with hereditary memory.

However, to the best of our knowledge, there are few results on Volterra diffusion equations with the CaputoFabrizio derivative (problem (1.1)). Our first main goal is to present global results on the whole space $\mathbb{R}^{N}$ under a global Lipschitz condition on the source term. The second goal is to prove the existence and uniqueness of mild solutions and present a blow-up alternative for mild solutions of problem (1.1) under a locally Lipschitz condition on $G$ and $\varphi$.

The paper is organized as follows. In Section 2, we introduce some important vector spaces, Lemma's, preliminaries, and the definition of mild solutions of (1.1). In Section 3, we prove global existence for the mild solution of problem (1.1) when $D=\mathbb{R}^{N}$, with globally Lipschitz source terms. Finally, in the last section, a local 
well-posed result will be given for problem (1.1) with locally Lipschitz condition when $D$ is an open bounded domain with smooth boundary in $\mathbb{R}^{N}$.

\section{Preliminary MATERial}

Assume that $D$ is a bounded domain whose boundary is sufficiently smooth. We consider the following spectral problem for the Laplace operator $\Delta$

$$
\begin{cases}-\Delta \psi_{j}(x)=\lambda_{j} \psi_{j}(x) & \text { in } D \\ \psi_{j}(0)=0 & \text { on } \partial D\end{cases}
$$

which admits a family of eigenvalues $0<\lambda_{1} \leq \lambda_{2} \leq \lambda_{3} \leq \cdots \leq \lambda_{j} \cdots \nearrow \infty$ and a corresponding family of eigenvectors $\left\{\psi_{j}\right\}_{j \in \mathbb{N}}$.

Let $\left(B,\|\cdot\|_{B}\right)$ be a Banach space, then, we denote by $L^{p}(0, T ; B)$ the Banach space of measurable functions $f:(0, T) \rightarrow B$ eqquiped with the norm

$$
\|f\|_{L^{p}(0, T ; B)}=\left(\int_{0}^{T}\|f(t)\|_{B}^{p} \mathrm{~d} t\right)^{\frac{1}{p}}, \quad \text { for } 1 \leq p<\infty
$$

and

$$
\|f\|_{L^{\infty}(0, T ; B)}=\underset{t \in(0, T)}{\operatorname{ess} \sup }\|f(t)\|_{B}, \quad \text { for } p=\infty
$$

The space of all $k$ time's derivative continuous functions $C^{k}([0, T] ; B), k \in \mathbb{N}$ is a Banach space with the norm

$$
\|f\|_{C^{k}([0, T] ; B)}=\sum_{i=1}^{k} \sup _{t \in[0, T]}\left\|f^{i}(t)\right\|_{B} .
$$

We denote by $\mathbb{X}^{h, \mu}((0, T] ; B)$, the subspace of all functions $f$ in $C([0, T] ; B)$ such that

$$
\|f\|_{\mathbb{X}^{h, \mu}((0, T] ; B)}:=\sup _{0<t \leq T} t^{h} e^{-\mu t}\|f(t)\|_{B}<\infty .
$$

Assume that $D$ is a bounded domain whose boundary is sufficiently smooth. For any $\sigma>0$, we define the fractional Hilbert scale space by

$$
\mathcal{H}^{\sigma}(D)=\left\{f \in L^{2}(D):\|f\|_{\mathcal{H}^{\sigma}(D)}^{2}=\sum_{j=1}^{\infty} \lambda_{j}^{2 \sigma}\left\langle f, \psi_{j}\right\rangle_{L^{2}(D)}^{2}<\infty\right\}
$$

and the Hilbert space $\mathcal{H}^{-\sigma}(D)$ with the norm

$$
\|f\|_{\mathcal{H}^{-\sigma}(D)}=\sqrt{\sum_{j=1}^{\infty} \lambda_{j}^{-2 \sigma}\left\langle f, \psi_{j}\right\rangle_{*}^{2}}
$$

which is the duality space of $\mathcal{H}^{\sigma}(D)$. The notation $\langle\cdot, \cdot\rangle_{*}$ denotes the dual inner product between $\mathcal{H}^{-\sigma}(D)$ and $\mathcal{H}^{\sigma}(D)$. 
Lemma 2.1. (see [15]) The Laplace transform of the Caputo-Fabrizio derivative is given by

$$
\mathscr{L}\left\{C F \mathscr{D}_{t}^{\alpha} w(t)\right\}(m)=\frac{m \mathscr{L}\{f\}(m)-f(0)}{m+\alpha(1-m)}, \quad m>0 .
$$

Definition 2.2. Let $\alpha \Delta[(1-\alpha) \Delta-I]^{-1}$ be the infinitesimal generator of an analytic semigroup $\{T(t), t \geq 0\}$. Then, the formula of the mild solution for problem (1.1) is given by

$$
\begin{aligned}
u(t)=\mathcal{A} T(\alpha t) u_{0} & +\int_{0}^{t} \alpha \mathcal{A}^{2} T(\alpha(t-z)) G(u(z)) \mathrm{d} z \\
& +\int_{0}^{t} \alpha \mathcal{A}^{2} T(\alpha(t-z)) \int_{0}^{z} \varphi(z-s, u(s)) \mathrm{d} s \mathrm{~d} z,
\end{aligned}
$$

where we set $\mathcal{A}=[I-\Delta(1-\alpha)]^{-1}$.

Lemma 2.3. (see [14])Assume that $D \equiv \mathbb{R}^{N}$. Let $1 \leq p \leq q$ and $w \in L^{p}(D) \cap L^{q}(D)$. Then, there exists a constant $C$ such that

$$
\begin{aligned}
& \|T(t) w\|_{L^{q}(D)} \leq C(1+t)^{\frac{N}{2}\left(\frac{1}{q}-\frac{1}{p}\right)}\|w\|_{L^{p}(D)}+C e^{-t}\|w\|_{L^{q}(D)}, \quad t \geq 0 . \\
& \|\mathcal{A} w\|_{L^{q}(D)} \leq C\|w\|_{L^{q}(D)} .
\end{aligned}
$$

\section{Result on the Whole SPACE $\mathbb{R}^{N}$ With GLOBAlly LiPsChitZ SOURCE TERM}

In this section, we consider the existence and uniqueness of mild solution for problem (1.1) when $D \equiv \mathbb{R}^{N}$ under the following assumptions for the source terms

$$
\left\{\begin{array}{l}
G(0)=0 \\
|G(a)-G(b)| \leq L|a-b|, \quad \forall a, b \in \mathbb{R}
\end{array}\right.
$$

and

$$
\left\{\begin{array}{l}
\varphi(0)=0 \\
|\varphi(a)-\varphi(b)| \leq L|a-b|, \quad \forall a, b \in \mathbb{R}
\end{array}\right.
$$

where $L$ is not dependent on $a, b$.

Lemma 3.1. (see [19]) Let $h>0$ and $m, n>-1$ such that $m+n>-1$. Then, we have the following limit result

$$
\sup _{t \in[0, T]} t^{h} \int_{0}^{1} s^{m}(1-s)^{n} e^{-\mu t(1-s)} \mathrm{d} s \stackrel{\mu \longrightarrow \infty}{\longrightarrow} 0 .
$$

Theorem 3.2. Let $1 \leq p \leq q$ such that

$$
\frac{1}{p}-\frac{1}{q}<\frac{1}{N}
$$


and

$$
h \in\left(\frac{N(q-p)}{2 p q}, 1+\frac{N(p-q)}{2 p q}\right)
$$

Assume that $u_{0} \in Z=L^{p}(D) \cap L^{q}(D)$ and $G, \varphi$ satisfy (3.1), (3.2), respectively. Then, for any $T>0$, problem (1.1) has a unique mild solution in $\mathbb{X}^{h, \mu}((0, T] ; Z)$.

Proof. The proof begins by defining a mapping $J: \mathbb{X}^{h, \mu}((0, T] ; Z) \longrightarrow \mathbb{X}^{h, \mu}((0, T] ; Z)$ as follows

$$
\begin{aligned}
J w(t)=\mathcal{A} T(\alpha t) u_{0} & +\int_{0}^{t} \alpha \mathcal{A}^{2} T(\alpha(t-z)) G(w(z)) \mathrm{d} z \\
& +\int_{0}^{t} \alpha \mathcal{A}^{2} T(\alpha(t-z)) \int_{0}^{z} \varphi(z-s, w(s)) \mathrm{d} s \mathrm{~d} z .
\end{aligned}
$$

We first use Lemma 2.8 and the inequality $e^{-r} \leq c_{\theta} r^{-\theta}(r>0,0<\theta<1)$ to obtain

$$
\begin{aligned}
\left\|\mathcal{A} T(\alpha t) u_{0}\right\|_{L^{q}(D)} & \leq C^{2}(1+\alpha t)^{\frac{N}{2}\left(\frac{1}{q}-\frac{1}{p}\right)}\left\|u_{0}\right\|_{L^{p}(D)}+C^{2} e^{-\alpha t}\left\|u_{0}\right\|_{L^{q}(D)} \\
& \leq C^{2}(1+\alpha t)^{\frac{N}{2}\left(\frac{1}{q}-\frac{1}{p}\right)}\left\|u_{0}\right\|_{L^{p}(D)}+C^{2} c_{\theta} \alpha^{-\theta} t^{-\theta}\left\|u_{0}\right\|_{L^{q}(D)} .
\end{aligned}
$$

For $h>\frac{N}{2}\left(\frac{1}{p}-\frac{1}{q}\right)$, choosing $\theta=\frac{N}{2}\left(\frac{1}{p}-\frac{1}{q}\right)<1$, we deduce that

$$
\begin{aligned}
t^{h} e^{-\mu t}\left\|\mathcal{A} T(\alpha t) u_{0}\right\|_{L^{q}(D)} & \leq C^{2} \alpha^{\frac{N}{2}\left(\frac{1}{q}-\frac{1}{p}\right)} T^{h+\frac{N}{2}\left(\frac{1}{q}-\frac{1}{p}\right)}\left(\left\|u_{0}\right\|_{L^{p}(D)}+c_{\theta}\left\|u_{0}\right\|_{L^{q}(D)}\right) \\
& \leq \max \left\{1, c_{\theta}\right\} C^{2} T^{h+\frac{N}{2}\left(\frac{1}{q}-\frac{1}{p}\right)}\left\|u_{0}\right\|_{Z} .
\end{aligned}
$$

Next, we will deal with the remain term $J w(t)-\mathcal{A} T(\alpha t) u_{0}$. To this end, for any $w, v \in \mathbb{X}^{h, \mu}((0, T] ; Z)$, we will consider the following equation

$$
J w(t)-J v(t)=\mathcal{I}_{1}+\mathcal{I}_{2},
$$

where

$$
\begin{aligned}
& \mathcal{I}_{1}=\alpha \int_{0}^{t} \mathcal{A}^{2} T(\alpha(t-z))[G(w(z))-G(v(z))] \mathrm{d} z, \\
& \mathcal{I}_{2}=\alpha \int_{0}^{t} \mathcal{A}^{2} T(\alpha(t-z)) \int_{0}^{z}[\varphi(z-s, w(s))-\varphi(z-s, v(s))] \mathrm{d} s \mathrm{~d} z .
\end{aligned}
$$

Using the same calculation ideas as in (3.5), we see that

$$
\begin{aligned}
& \left\|\mathcal{A}^{2} T(\alpha(t-z))[G(w(z))-G(v(z))]\right\|_{L^{q}(D)} \\
& \leq \frac{C^{3}}{(1+\alpha(t-z))^{\frac{N}{2}\left(\frac{1}{p}-\frac{1}{q}\right)}}\|G(w(z))-G(v(z))\|_{L^{p}(D)}+\frac{C^{3}}{e^{\alpha(t-z)}}\|G(w(z))-G(v(z))\|_{L^{q}(D)} \\
& \leq \frac{C^{3}}{(\alpha(t-z))^{\frac{N}{2}\left(\frac{1}{p}-\frac{1}{q}\right)}}\|G(w(z))-G(v(z))\|_{L^{p}(D)}+\frac{C^{3} c_{\theta}}{\alpha^{\theta}(t-z)^{\theta}}\|G(w(z))-G(v(z))\|_{L^{q}(D)} .
\end{aligned}
$$


Then, it follows that

$$
\left\|\mathcal{I}_{1}\right\|_{L^{q}(D)} \leq M_{1} \int_{0}^{t}(t-z)^{\frac{N}{2}\left(\frac{1}{q}-\frac{1}{p}\right)}\|w(z)-v(z)\|_{Z} \mathrm{~d} z
$$

where $M_{1}=C^{3} L \alpha^{\frac{N}{2}\left(\frac{1}{q}-\frac{1}{p}\right)} \max \left\{1, c_{\theta}\right\}$. The above estimate gives us the one below

$$
\begin{aligned}
t^{h} e^{-\mu t}\left\|\mathcal{I}_{1}\right\|_{L^{q}(D)} & \leq M_{1} t^{h} e^{-\mu t} \int_{0}^{t}(t-z)^{\frac{N}{2}\left(\frac{1}{q}-\frac{1}{p}\right)}\|w(z)-v(z)\|_{Z} \mathrm{~d} z \\
& \leq M_{1} t^{h} \int_{0}^{t} z^{-h}(t-z)^{\frac{N}{2}\left(\frac{1}{q}-\frac{1}{p}\right)} e^{-\mu(t-z)} \mathrm{d} z\|w-v\|_{\mathbb{X}^{h, \mu}((0, T] ; Z)} .
\end{aligned}
$$

In Lemma 2.8, taking $p=q$ and setting $M_{2}=C^{3} L \alpha^{\frac{N}{2}\left(\frac{1}{q}-\frac{1}{p}\right)}\left(1+c_{\theta}\right)$, we have

$$
\begin{aligned}
t^{h} e^{-\mu t}\left\|\mathcal{I}_{1}\right\|_{L^{p}(D)} & \leq t^{h} e^{-\mu t} M_{2} \int_{0}^{t}(t-z)^{\frac{N}{2}\left(\frac{1}{q}-\frac{1}{p}\right)}\|w(z)-v(z)\|_{Z} \mathrm{~d} z \\
& \leq M_{2} t^{h} \int_{0}^{t} z^{-h}(t-z)^{\frac{N}{2}\left(\frac{1}{q}-\frac{1}{p}\right)} e^{-\mu(t-z)} \mathrm{d} z\|w-v\|_{\mathbb{X}^{h, \mu}((0, T] ; Z)} .
\end{aligned}
$$

These two estimates above lead us to the following result

$$
t^{h} e^{-\mu t}\left\|\mathcal{I}_{1}\right\|_{Z} \leq\left(M_{1}+M_{2}\right) t^{h} \int_{0}^{t} z^{-h}(t-z)^{\frac{N}{2}\left(\frac{1}{q}-\frac{1}{p}\right)} e^{-\mu(t-z)} \mathrm{d} z\|w-v\|_{\mathbb{X}^{h, \mu}((0, T] ; Z)} .
$$

By similar arguments, we also obtain

$$
\begin{aligned}
t^{h} e^{-\mu t}\left\|\mathcal{I}_{2}\right\|_{Z} & \leq\left(M_{1}+M_{2}\right) t^{h} e^{-\mu t} \int_{0}^{t}(t-z)^{\frac{N}{2}\left(\frac{1}{q}-\frac{1}{p}\right)} \int_{0}^{z}\|w(s)-v(s)\|_{Z} \mathrm{~d} s \mathrm{~d} z \\
& \leq\left(M_{1}+M_{2}\right) t^{h} \int_{0}^{t}(t-z)^{\frac{N}{2}\left(\frac{1}{q}-\frac{1}{p}\right)} e^{-\mu(t-z)} \int_{0}^{z} s^{-h} \mathrm{~d} s \mathrm{~d} z\|w-v\|_{\mathbb{X}^{h, \mu}((0, T] ; Z)} \\
& \leq \frac{\left(M_{1}+M_{2}\right) T}{1-h} t^{h} \int_{0}^{t} z^{-h}(t-z)^{\frac{N}{2}\left(\frac{1}{q}-\frac{1}{p}\right)} e^{-\mu(t-z)} \mathrm{d} z\|w-v\|_{\mathbb{X}^{h, \mu}((0, T] ; Z)} .
\end{aligned}
$$

On account of the results stated above, by choosing $v=0$, it's easily check that if we take $w$ in $\mathbb{X}^{h, \mu}((0, T] ; Z)$, $J w$ will be in $\mathbb{X}^{h, \mu}((0, T] ; Z)$. In addition, the standard smooth effect of the semigroup $T(t)$ will ensure the continuity of $J$ on $(0, T]$. Therefore, we conclude that, $J$ is invariant on $\mathbb{X}^{h, \mu}((0, T] ; Z)$.

In the other hand, from the assumptions on $p, q, h$, we can easily deduce that

$$
\left\{\begin{array}{l}
-h>-1, \\
\frac{N}{2}\left(\frac{1}{q}-\frac{1}{p}\right)>-1, \\
-h+\frac{N}{2}\left(\frac{1}{q}-\frac{1}{p}\right)>-1, \\
h+\frac{N}{2}\left(\frac{1}{q}-\frac{1}{p}\right)>0 .
\end{array}\right.
$$


Lemma 3.1 is now applied to yield that

$$
\lim _{\mu \rightarrow \infty} \mathcal{Q}(\mu):=\lim _{\mu \rightarrow \infty}\left[\sup _{0<t \leq T} t^{h+\frac{N}{2}\left(\frac{1}{q}-\frac{1}{p}\right)} \int_{0}^{1} z^{-h}(1-z)^{\frac{N}{2}\left(\frac{1}{q}-\frac{1}{p}\right)} e^{-\mu t(1-z)} \mathrm{d} z\right]=0 .
$$

Combining (3.7), (3.14), (3.15), we have

$$
t^{h} e^{-\mu t}\|J w(t)-J v(t)\|_{Z} \leq M\left(1+\frac{T}{1-h}\right) \mathcal{Q}(\mu)\|w-v\|_{\mathbb{X}^{h, \mu}((0, T] ; Z)} .
$$

From (3.16), there exists a sufficiently large $\mu_{0}$ such that

$$
M\left(1+\frac{T}{1-h}\right) \mathcal{Q}\left(\mu_{0}\right)<1
$$

Consequently, $J$ is a contraction on $\mathbb{X}^{h, \mu}((0, T] ; Z)$. Now we conclude that problem (1.1) has a unique solution in $\mathbb{X}^{h, \mu}((0, T] ; Z)$ and the proof is completed.

\section{Problem (1.1) UNDER LOCAlly Lipschitz SOURCE TERM}

Throughout this section, we suppose that $D \subset \mathbb{R}^{N}$ is an open bounded domain with smooth boundary and our solution will vanish on the boundary of $D$. Furthermore, we consider the following assumptions for the source terms.

Let $\nu<\sigma<\nu+2$ and $m, n>1$. Suppose that for any $a, b \in \mathbb{R}$, functions $G, \varphi$ satisfy

$$
\left\{\begin{array}{l}
\|G(a)-G(b)\|_{\mathcal{H}^{\nu}(D)} \leq L_{G}\left(1+\|a\|_{\mathcal{H}^{\sigma}(D)}^{m-1}+\|b\|_{\mathcal{H}^{\sigma}(D)}^{m-1}\right)\|a-b\|_{\mathcal{H}^{\sigma}(D)} \\
\|G(a)\|_{\mathcal{H}^{\nu}(D)} \leq L_{G}\left(\|a\|_{\mathcal{H}^{\sigma}(D)}^{m}+1\right)
\end{array}\right.
$$

and

$$
\left\{\begin{array}{l}
\|\varphi(a)-\varphi(b)\|_{\mathcal{H}^{\nu}(D)} \leq L_{\varphi}\left(1+\|a\|_{\mathcal{H}^{\sigma}(D)}^{n-1}+\|b\|_{\mathcal{H}^{\sigma}(D)}^{n-1}\right)\|a-b\|_{\mathcal{H}^{\sigma}(D)} \\
\|\varphi(a)\|_{\mathcal{H}^{\nu}(D)} \leq L_{\varphi}\left(\|a\|_{\mathcal{H}^{\sigma}(D)}^{n}+1\right)
\end{array}\right.
$$

To deal with the initial value problem with bounded domain, we will rewrite (2.7) in the form of Fourier series as follows (see [39])

$$
u(t)=\mathcal{S}_{0}(t) u_{0}+\int_{0}^{t} \mathcal{S}(t-z) G(u(z)) \mathrm{d} z+\int_{0}^{t} \mathcal{S}(t-z) \int_{0}^{z} \varphi(z-s, u(s)) \mathrm{d} s \mathrm{~d} z
$$

where

$$
\mathcal{S}_{0}(t) f(x):=\sum_{j=1}^{\infty} \frac{1}{1+\lambda_{j}(1-\alpha)} \exp \left(\frac{-\lambda_{j} \alpha t}{1+\lambda_{j}(1-\alpha)}\right)\left\langle f, \psi_{j}\right\rangle_{L^{2}(D)} \psi_{j}(x)
$$

and

$$
\mathcal{S}(t) f(x):=\sum_{j=1}^{\infty} \frac{\alpha}{\left[1+\lambda_{j}(1-\alpha)\right]^{2}} \exp \left(\frac{-\lambda_{j} \alpha t}{1+\lambda_{j}(1-\alpha)}\right)\left\langle f, \psi_{j}\right\rangle_{L^{2}(D)} \psi_{j}(x)
$$




\subsection{Local existence and uniqueness of the mild solution}

Lemma 4.1. Let $\mathcal{K}, T_{0}$ be positive constants. Assume that $u \in C\left(\left[0, T_{0}\right] ; \mathcal{H}^{\sigma}(D)\right)$ and $f \in \mathcal{H}^{\sigma}(D)$ such that $\sup _{t \in\left[0, T_{0}\right]}\|u(t)-f\|_{\mathcal{H}^{\sigma}(D)} \leq \mathcal{K}$, then for $0 \leq t_{1}<t_{2} \leq T_{0}$, we have the following estimates

$$
\left\|\int_{t_{1}}^{t_{2}} \mathcal{S}\left(t_{2}-z\right) G(u(z)) \mathrm{d} z\right\|_{\mathcal{H}^{\sigma}(D)} \leq \frac{L_{G}\left(t_{2}-t_{1}\right)}{(1-\alpha)^{2}}\left[\left(\|f\|_{\mathcal{H}^{\sigma}(D)}+\mathcal{K}\right)^{m}+1\right]
$$

and

$$
\left\|\int_{t_{1}}^{t_{2}} \mathcal{S}(t-z) \int_{0}^{z} \varphi(z-s, u(s)) \mathrm{d} s \mathrm{~d} z\right\|_{\mathcal{H}^{\sigma}(D)} \leq \frac{L_{\varphi}\left(t_{2}-t_{1}\right)}{T_{0}^{-1}(1-\alpha)^{2}}\left[\left(\|f\|_{\mathcal{H}^{\sigma}(D)}+\mathcal{K}\right)^{n}+1\right]
$$

Proof. Using the triangle inequality, we have

$$
\|u(t)\|_{\mathcal{H}^{\sigma}(D)} \leq\|f\|_{\mathcal{H}^{\sigma}(D)}+\sup _{0 \leq t \leq T_{0}}\|u(t)-f\|_{\mathcal{H}^{\sigma}(D)} \leq\|f\|_{\mathcal{H}^{\sigma}(D)}+\mathcal{K}
$$

Thanks to the Parseval identity, the following estimate holds

$$
\begin{aligned}
& \left\|\mathcal{S}\left(t_{2}-z\right) G(u(z))\right\|_{\mathcal{H}^{\sigma}(D)}^{2} \\
& \leq \sum_{j=1}^{\infty} \frac{\lambda_{j}^{2 p}}{\left(1+\lambda_{j}(1-\alpha)\right)^{4}} \exp \left(-\frac{2 \alpha \lambda_{j}\left(t_{2}-z\right)}{1+\lambda_{j}(1-\alpha)}\right)\left\langle G(u(z)), \psi_{j}\right\rangle_{L^{2}(\Omega)}^{2} \\
& \leq \frac{1}{(1-\alpha)^{4}} \sum_{j=1}^{\infty} \lambda_{j}^{2 p-4}\left\langle G(u(z)), \psi_{j}\right\rangle_{L^{2}(\Omega)}^{2} \leq \frac{1}{(1-\alpha)^{4}}\|G(u(z))\|_{\mathcal{H}^{\nu}(D)}^{2} .
\end{aligned}
$$

The above result gives

$$
\begin{aligned}
& \left\|\int_{t_{1}}^{t_{2}} \mathcal{S}\left(t_{2}-z\right) G(u(z)) \mathrm{d} z\right\|_{\mathcal{H}^{\sigma}(D)} \leq \frac{L_{G}}{(1-\alpha)^{2}} \int_{t_{1}}^{t_{2}}\left(\|u(z)\|_{\mathcal{H}^{\sigma}(D)}^{m}+1\right) \mathrm{d} z \\
& \leq \frac{L_{G}}{(1-\alpha)^{2}}\left[\left(\|f\|_{\mathcal{H}^{\sigma}(D)}+\mathcal{K}\right)^{m}+1\right]\left(t_{2}-t_{1}\right) .
\end{aligned}
$$

Similarly, the second conclusion of this lemma can also be drawn

$$
\begin{aligned}
& \left\|\int_{t_{1}}^{t_{2}} \mathcal{S}(t-z) \int_{0}^{z} \varphi(z-s, u(s)) \mathrm{d} s \mathrm{~d} z\right\|_{\mathcal{H}^{\sigma}(D)} \\
& \quad \leq \frac{L_{\varphi}}{(1-\alpha)^{2}} \int_{t_{1}}^{t_{2}} \int_{0}^{z}\left(\|u(s)\|_{\mathcal{H}^{\sigma}(D)}^{n}+1\right) \mathrm{d} s \mathrm{~d} z \\
& \quad \leq \frac{t_{2} L_{\varphi}}{(1-\alpha)^{2}}\left[\left(\|f\|_{\mathcal{H}^{\sigma}(D)}+\mathcal{K}\right)^{n}+1\right]\left(t_{2}-t_{1}\right) .
\end{aligned}
$$

Theorem 4.2. Let $G$ and $\varphi$ sastify the locally Lipschitz condition (4.1), (4.2), respectively. Assume that $u_{0} \in$ $\mathcal{H}^{\sigma}(D)$. Then, there exists $T_{0}>0$ such that problem (1.1) has a unique mild solution in $C\left(\left[0, T_{0}\right] ; \mathcal{H}^{\sigma}(D)\right)$. 
Proof. Set $\widetilde{u_{0}}=\frac{u_{0}}{1+\lambda_{j}(1-\alpha)}$ and we can check immediately that $\widetilde{u_{0}} \in \mathcal{H}^{\sigma}(D)$. Next, for a fixed positive constant $\mathcal{K}$, we consider the following space

$$
\mathbb{A}:=\left\{u \in C\left(\left[0, T_{0}\right] ; \mathcal{H}^{\sigma}(D)\right):\left\|u(\cdot ; t)-\widetilde{u_{0}}\right\|_{\mathcal{H}^{\sigma}(D)} \leq \mathcal{K}, \forall t \in\left[0, T_{0}\right]\right\}
$$

and define the operator $J: \mathbb{A} \rightarrow \mathbb{A}$ by

$$
J w(t, x)=\mathcal{S}_{0}(t) u_{0}+\alpha \int_{0}^{t} \mathcal{S}(t-z) G(w(z)) \mathrm{d} z+\alpha \int_{0}^{t} \mathcal{S}(t-z) \int_{0}^{z} \varphi(z-s, w(s)) \mathrm{d} s \mathrm{~d} z .
$$

Note that $\mathbb{A}$ is a complete space with respect to the usual sup norm. For the purpose of using the Banach fixed point theorem, we first need to show that $J w \in C\left(\left[0, T_{0}\right] ; \mathcal{H}^{\sigma}(D)\right)$ for any $w \in C\left(\left[0, T_{0}\right] ; \mathcal{H}^{\sigma}(D)\right)$. Let $0<t \leq t+\varepsilon \leq T_{0}$. Using the inequality $\left|e^{-a}-e^{-b}\right| \leq|a-b|$, for $a, b>0$, we have

$$
\begin{aligned}
\left\|\left(\mathcal{S}_{0}(t+\varepsilon)-\mathcal{S}_{0}(t)\right) u_{0}\right\|_{\mathcal{H}^{\sigma}(D)} & \leq \sqrt{\frac{\alpha^{2} \varepsilon^{2}}{\lambda_{1}^{2}(1-\alpha)^{4}} \sum_{j=1}^{\infty} \lambda_{j}^{2 p}\left\langle u_{0}, e_{j}\right\rangle_{L^{2}(D)}^{2}} \\
& =\frac{\alpha \varepsilon}{\lambda_{1}(1-\alpha)^{2}}\left\|u_{0}\right\|_{\mathcal{H}^{\sigma}(D)} .
\end{aligned}
$$

and

$$
\begin{aligned}
& \left\|\int_{0}^{t}(\mathcal{S}(t+\varepsilon-z)-\mathcal{S}(t-z)) G(w(z)) \mathrm{d} z\right\|_{\mathcal{H}^{\sigma}(D)} \\
& \leq \frac{\alpha \varepsilon}{(1-\alpha)^{3}} \int_{0}^{t}\|G(w(z))\|_{\mathcal{H}^{\nu}(D)} \mathrm{d} z \leq\left[\left(\left\|\widetilde{u_{0}}\right\|_{\mathcal{H}^{\sigma}(D)}+\mathcal{K}\right)^{m}+1\right] \frac{\alpha \varepsilon T_{0} L_{G}}{(1-\alpha)^{3}} .
\end{aligned}
$$

In the other hand, it follows from Lemma 4.1 that

$$
\left\|\int_{t}^{t+\varepsilon} \mathcal{S}(t+\varepsilon-z) G(w(z)) \mathrm{d} z\right\|_{\mathcal{H}^{\sigma}(D)} \leq \frac{L_{G} \varepsilon}{(1-\alpha)^{2}}\left[\left(\left\|\widetilde{u_{0}}\right\|_{\mathcal{H}^{\sigma}(D)}+\mathcal{K}\right)^{m}+1\right] .
$$

By similar arguments, we also obtain

$$
\begin{aligned}
& \left\|\int_{t}^{t+\varepsilon} \mathcal{S}(t+\varepsilon-z) \int_{0}^{z} \varphi(z-s, w(s)) \mathrm{d} s \mathrm{~d} z\right\|_{\mathcal{H}^{\sigma}(D)} \\
& \quad \leq \frac{L_{\varphi} T_{0} \varepsilon}{(1-\alpha)^{2}}\left[\left(\left\|\widetilde{u_{0}}\right\|_{\mathcal{H}^{\sigma}(D)}+\mathcal{K}\right)^{n}+1\right]
\end{aligned}
$$

and

$$
\begin{aligned}
& \left\|\int_{0}^{t}(\mathcal{S}(t+\varepsilon-z)-\mathcal{S}(t-z)) \int_{0}^{z} \varphi(z-s, w(s)) \mathrm{d} s \mathrm{~d} z\right\|_{\mathcal{H}^{\sigma}(D)} \\
& \quad \leq\left[\left(\left\|\widetilde{u_{0}}\right\|_{\mathcal{H}^{\sigma}(D)}+\mathcal{K}\right)^{n}+1\right] \frac{\alpha \varepsilon T_{0}^{2} L_{\varphi}}{(1-\alpha)^{3}} .
\end{aligned}
$$


Hence, if $w$ belongs to $C\left(\left[0, T_{0}\right] ; \mathcal{H}^{\sigma}(D)\right)$, then, $J w$ is in $C\left(\left[0, T_{0}\right] ; \mathcal{H}^{\sigma}(D)\right)$. Next, we set

$$
\mathcal{M}=\max \left\{\left(\frac{\left\|u_{0}\right\|_{\mathcal{H}^{\sigma}(D)}}{\lambda_{1}(1-\alpha)}+\mathcal{K}\right)^{m},\left(\frac{\left\|u_{0}\right\|_{\mathcal{H}^{\sigma}(D)}}{\lambda_{1}(1-\alpha)}+\mathcal{K}\right)^{n}\right\}
$$

and choose a sufficiently small $T_{0}$ such that

$$
\left\{\begin{array}{l}
\frac{\alpha T_{0} \lambda_{1}^{-1}\left\|u_{0}\right\|_{\mathcal{H}^{\sigma}(D)}}{(1-\alpha)^{2}}+\frac{\left(L_{G} T_{0}+L_{\varphi} T_{0}^{2}\right)(\mathcal{M}+1)}{(1-\alpha)^{2}} \leq \mathcal{K}, \\
\left(L_{G} T_{0}+L_{\varphi} T_{0}^{2}\right)<\frac{(1-\alpha)^{2}}{1+2 \mathcal{M}} .
\end{array}\right.
$$

Using the inequality $1-e^{r} \leq r$, we have

$$
\begin{aligned}
\left\|\mathcal{S}_{0}(t) u_{0}-\widetilde{u_{0}}\right\|_{\mathcal{H}^{\sigma}(D)}^{2} & \leq \sum_{j=1}^{\infty} \frac{\lambda_{j}^{2 \sigma}}{\left(1+\lambda_{j}(1-\alpha)\right)^{2}}\left[\exp \left(\frac{-\alpha \lambda_{j} t}{1+\lambda_{j}(1-\alpha)}\right)-1\right]^{2}\left\langle u_{0}, \psi_{j}\right\rangle_{L^{2}(D)}^{2} \\
& \leq \frac{(\alpha t)^{2}}{\lambda_{1}^{2}(1-\alpha)^{4}} \sum_{j=1}^{\infty} \lambda_{j}^{2 \sigma}\left\langle u_{0}, \psi_{j}\right\rangle_{L^{2}(D)}^{2} .
\end{aligned}
$$

Let $w \in \mathbb{A}$. From Lemma 4.1 and the above estimate, we get

$$
\begin{aligned}
\left\|J w(t)-\widetilde{u_{0}}\right\|_{\mathcal{H}^{\sigma}(D)} \leq & \left\|\mathcal{S}_{0}(t) u_{0}-\widetilde{u_{0}}\right\|_{\mathcal{H}^{\sigma}(D)} \\
& +\left\|\int_{0}^{t} \mathcal{S}(t-z) G(w(z)) \mathrm{d} z\right\|_{\mathcal{H}^{\sigma}(D)} \\
& +\left\|\int_{0}^{t} \mathcal{S}(t-z) \int_{0}^{z} \varphi(z-s, w(s)) \mathrm{d} s \mathrm{~d} z\right\|_{\mathcal{H}^{\sigma}(D)} \\
\leq & \frac{\alpha T_{0}}{\lambda_{1}(1-\alpha)^{2}}\left\|u_{0}\right\|_{\mathcal{H}^{\sigma}(D)}+\frac{L_{G} T_{0}+L_{\varphi} T_{0}^{2}}{(1-\alpha)^{2}}(\mathcal{M}+1) .
\end{aligned}
$$

Thus, $J w \in \mathbb{A}$. Now, we show that $J$ is a contraction. Let $v, w \in \mathbb{A}$, for any $t \in\left[0, T_{0}\right]$, we have

$$
\begin{aligned}
\|J w(t)-J v(t)\|_{\mathcal{H}^{\sigma}(D)} \leq & \int_{0}^{t}\|\mathcal{S}(t-z)(G(w(z))-G(v(z)))\|_{\mathcal{H}^{\sigma}(D)} \mathrm{d} z \\
& +\int_{0}^{t}\left\|\mathcal{S}(t-z) \int_{0}^{z}(\varphi(z-s, w(s))-\varphi(z-s, v(s))) \mathrm{d} s\right\|_{\mathcal{H}^{\sigma}(D)} \mathrm{d} z .
\end{aligned}
$$

Note for the first term (one can apply a similar argument for the second term)

$$
\begin{aligned}
& \int_{0}^{t}\|\mathcal{S}(t-z)(G(w(z))-G(v(z)))\|_{\mathcal{H}^{\sigma}(D)} \mathrm{d} z \\
& \quad \leq \frac{1}{(1-\alpha)^{2}} \int_{0}^{t}\|G(w(\cdot, z))-G(v(\cdot, z))\|_{\mathcal{H}^{\nu}(D)} \mathrm{d} z \\
& \quad \leq \frac{L_{G}}{(1-\alpha)^{2}} \int_{0}^{t}\left(1+\|v\|_{\mathcal{H}^{\sigma}(D)}^{m-1}+\|w\|_{\mathcal{H}^{\sigma}(D)}^{m-1}\right)\|v-w\|_{\mathcal{H}^{\sigma}(D)} \mathrm{d} z
\end{aligned}
$$




$$
\begin{aligned}
& \leq \frac{L_{G}}{(1-\alpha)^{2}} \int_{0}^{t}\|v-w\|_{\mathcal{H}^{\sigma}(D)}\left[1+2\left(\mathcal{K}+\left\|\widetilde{u_{0}}\right\|_{\mathcal{H}^{\sigma}(D)}\right)^{m-1}\right] \mathrm{d} z \\
& \leq \frac{L_{G} T_{0}}{(1-\alpha)^{2}}(1+2 \mathcal{M})\|v-w\|_{C\left(\left[0, T_{0}\right] ; \mathcal{H}^{\sigma}(D)\right)} .
\end{aligned}
$$

Thus

$$
\|J w(t)-J v(t)\|_{C\left(\left[0, T_{0}\right] ; \mathcal{H}^{\sigma}(D)\right)} \leq \frac{\left(L_{G} T_{0}+L_{\varphi} T_{0}^{2}\right)}{(1-\alpha)^{2}}(1+2 \mathcal{M})\|v-w\|_{C\left(\left[0, T_{0}\right] ; \mathcal{H}^{\sigma}(D)\right)} .
$$

From this point of view, we can conclude that $J$ is a contraction on $\mathbb{A}$. Therefore, there exists a unique solution $u$ of (4.10) and the theorem is proved.

\subsection{Continuation and blow-up alternative}

In this subsection, we present a continuation result and a blow-up alternative for the mild solution of problem (1.1).

Theorem 4.3. Assume that $G$ sastifies (4.1) and $\varphi$ satisfies (4.2). Then, the mild solution $u$ of problem (1.1) on $\left[0, T_{0}\right]$ can be extended to $\left[0, T_{0}+h\right]$, for some $h>0$.

Proof. Consider the following space

$$
\begin{array}{r}
\mathbb{B}=\left\{v \in C\left(\left[0, T_{0}+h\right], \mathcal{H}^{\sigma}(D)\right): v(t)=u(t), \forall t \in\left[0, T_{0}\right]\right. \\
\left.\quad \text { and }\left\|v(t)-u\left(T_{0}\right)\right\|_{\mathcal{H}^{\sigma}(D)} \leq \mathcal{M}, \forall t \in\left[T_{0}, T_{0}+h\right]\right\} .
\end{array}
$$

It's obvious that $\mathbb{B}$ is a complete space. Define a mapping $\mathscr{J}: \mathbb{B} \rightarrow \mathbb{B}$ by

$$
\mathscr{J} w(t, x)=\mathcal{S}_{0}(t) u_{0}+\alpha \int_{0}^{t} \mathcal{S}(t-z) G(w(z)) \mathrm{d} z+\alpha \int_{0}^{t} \mathcal{S}(t-z) \int_{0}^{z} \varphi(z-s, w(s)) \mathrm{d} s \mathrm{~d} z .
$$

Let $v \in \mathbb{B}$. If $t \in\left[0, T_{0}\right]$ we have $\mathscr{J} w(t)=\mathscr{J} u(t)=u(t)$. If $t \in\left[T_{0}, T_{0}+h\right]$, then

$$
\begin{aligned}
\left\|\mathscr{J} v(t)-u\left(T_{0}\right)\right\|_{\mathcal{H}^{\sigma}(D)} \leq & \left\|\left(\mathcal{S}_{0}(t)-\mathcal{S}_{0}\left(T_{0}\right)\right) u_{0}\right\|_{\mathcal{H}^{\sigma}(D)} \\
& +\int_{T_{0}}^{t}\|\mathcal{S}(t-z) G(v(z))\|_{\mathcal{H}^{\sigma}(D)} \mathrm{d} z \\
& +\int_{T_{0}}^{t}\left\|\mathcal{S}(t-z) \int_{0}^{z} \varphi(z-s, v(s)) \mathrm{d} s\right\|_{\mathcal{H}^{\sigma}(D)} \mathrm{d} z \\
& +\int_{0}^{T_{0}}\left\|\left(\mathcal{S}(t-z)-\mathcal{S}\left(T_{0}-z\right)\right) G(u(z))\right\|_{\mathcal{H}^{\sigma}(D)} \mathrm{d} z \\
& +\int_{0}^{T_{0}}\left\|\left(\mathcal{S}(t-z)-\mathcal{S}\left(T_{0}-z\right)\right) \int_{0}^{z} \varphi(z-s, u(s)) \mathrm{d} s\right\|_{\mathcal{H}^{\sigma}(D)} \mathrm{d} z .
\end{aligned}
$$

Using some arguments as in the proof of the previous theorem, we have

\section{Claim 1.}

$$
\left\|\left(\mathcal{S}_{0}(t)-\mathcal{S}_{0}\left(T_{0}\right)\right) u_{0}\right\|_{\mathcal{H}^{\sigma}(D)} \leq \frac{\alpha\left(t-T_{0}\right)}{\lambda_{1}(1-\alpha)^{2}}\left\|u_{0}\right\|_{\mathcal{H}^{\sigma}(D)}
$$




$$
\leq \frac{h \alpha}{\lambda_{1}(1-\alpha)^{2}}\left\|u_{0}\right\|_{\mathcal{H}^{\sigma}(D)}
$$

Claim 2.

$$
\int_{T_{0}}^{t}\|\mathcal{S}(t-z) G(v(z))\|_{\mathcal{H}^{\sigma}(D)} \mathrm{d} z \leq \frac{h L_{G}}{(1-\alpha)^{2}}\left[\left(\left\|u\left(T_{0}\right)\right\|_{\mathcal{H}^{\sigma}(D)}+\mathcal{M}\right)^{m}+1\right] .
$$

Claim 3.

$$
\int_{0}^{T_{0}}\left\|\left(\mathcal{S}(t-z)-\mathcal{S}\left(T_{0}-z\right)\right) G(u(z))\right\|_{\mathcal{H}^{\sigma}(D)} \mathrm{d} z \leq \frac{h L_{G} T_{0}}{(1-\alpha)^{2}}\left(\|u\|_{\left(C\left[0, T_{0}\right], \mathcal{H}^{\sigma}(D)\right)}^{m}+1\right) .
$$

\section{Claim 4.}

$$
\int_{T_{0}}^{t}\left\|\mathcal{S}(t-z) \int_{0}^{z} \varphi(z-s, v(s)) \mathrm{d} s\right\|_{\mathcal{H}^{\sigma}(D)} \mathrm{d} z \leq \frac{h T_{0} L_{\varphi}}{(1-\alpha)^{2}}\left[\left(\left\|u\left(T_{0}\right)\right\|_{\mathcal{H}^{\sigma}(D)}+\mathcal{M}\right)^{n}+1\right] .
$$

\section{Claim 5.}

$$
\begin{aligned}
\int_{0}^{T_{0}} \|(\mathcal{S}(t-z)-\mathcal{S} & \left.\left(T_{0}-z\right)\right) \int_{0}^{z} \varphi(z-s, u(s)) \mathrm{d} s \|_{\mathcal{H}^{\sigma}(D)} \mathrm{d} z \\
\leq & \frac{h T_{0} L_{\varphi} T_{0}}{(1-\alpha)^{2}}\left(\|u\|_{\left(C\left[0, T_{0}\right], \mathcal{H}^{\sigma}(D)\right)}^{n}+1\right) .
\end{aligned}
$$

Combining the above claims, we can choose some $h>0$ and $\mathcal{M}>0$ such that the right hand side of the above estimate is less than $\mathcal{M}$, so $\mathscr{J} v$ is in $\mathbb{B}$.

Next, take $v, w \in \mathbb{B}$. Then

$$
\begin{aligned}
\| \mathscr{J} v(t) & -\mathscr{J} w(t) \|_{\mathcal{H}^{\sigma}(D)} \\
\leq & \int_{T_{0}}^{t}\|\mathcal{S}(t-z)(G(v(z))-G(w(z)))\|_{\mathcal{H}^{\sigma}(D)} \mathrm{d} z \\
& \quad+\int_{T_{0}}^{t} \| \mathcal{S}(t-z) \int_{0}^{z}(\varphi(z-s, v(s))-\varphi(z-s, w(s))) \mathrm{d} s \\
\leq & \frac{L_{G}}{(1-\alpha)^{2}} \int_{T_{0}}^{t}\left(1+\|v\|_{\mathcal{H}^{\sigma}(D)}^{p-1}+\|w\|_{\mathcal{H}^{\sigma}(D)}^{p-1}\right)\|v-w\|_{\mathcal{H}^{\sigma}(D)} \mathrm{d} z . \\
& +\frac{\left(L_{\varphi} T_{0}\right.}{(1-\alpha)^{2}} \int_{T_{0}}^{t}\left(1+\|v\|_{\mathcal{H}^{\sigma}(D)}^{p-1}+\|w\|_{\mathcal{H}^{\sigma}(D)}^{p-1}\right)\|v-w\|_{\mathcal{H}^{\sigma}(D)} \mathrm{d} z .
\end{aligned}
$$

Since $v, w \in \mathbb{B}$, for $t \in\left[T_{0}, T_{0}+h\right]$ we have

$$
\|v\|_{\mathcal{H}^{\sigma}(D)}^{p-1}+\|w\|_{\mathcal{H}^{\sigma}(D)}^{p-1} \leq 2\left(\left\|u\left(T_{0}\right)\right\|_{\mathcal{H}^{\sigma}(D)}+\mathcal{M}\right)^{p-1} .
$$

Thus

$$
\|\mathscr{J} v(t)-\mathscr{J} w(t)\|_{\mathcal{H}^{\sigma}(D)}
$$




$$
\begin{aligned}
& \leq \frac{h L_{G}}{(1-\alpha)^{2}}\left[1+2\left(\left\|u\left(T_{0}\right)\right\|_{\mathcal{H}^{\sigma}(D)}+\mathcal{M}\right)^{p-1}\right]\|v-w\|_{C\left(\left[0, T_{0}+h\right], \mathcal{H}^{\sigma}(D)\right)} \\
& \leq \frac{h L_{\varphi} T_{0}}{(1-\alpha)^{2}}\left[1+2\left(\left\|u\left(T_{0}\right)\right\|_{\mathcal{H}^{\sigma}(D)}+\mathcal{M}\right)^{p-1}\right]\|v-w\|_{C\left(\left[0, T_{0}+h\right], \mathcal{H}^{\sigma}(D)\right)}
\end{aligned}
$$

We can find a $C_{h}<1$ (depending on h) such that

$$
\|\mathscr{J} v(t)-\mathscr{J} w(t)\|_{C\left(\left[0, T_{0}+h\right], \mathcal{H}^{\sigma}(D)\right)} \leq C_{h}\|v-w\|_{C\left(\left[0, T_{0}+h\right], \mathcal{H}^{\sigma}(D)\right)}
$$

Then $\mathscr{J}$ is a contraction on $\mathbb{B}$. Now apply the Banach Fixed Point Theorem to get the desired result.

Theorem 4.4. Assume that $G, \varphi$ satisfy (4.1) and (4.2). Let $u$ be the mild solution of problem (1.1) defined on $\left[0, T_{\max }\right)$, where $T_{\max }$ is the maximal time of existence of $u$. Then we have

$$
T_{\max }=\infty \quad \text { or } \lim _{t \rightarrow T_{\max }^{-}}\|u(\cdot, t)\|_{\mathcal{H}^{\sigma}(D)}=\infty
$$

Proof. Suppose that $T_{\max }<\infty$ and there exists a constant $\Im>0$ such that

$$
\max \left\{\left\|u_{0}\right\|_{\mathcal{H}^{\sigma-2}(D)}, \sup _{0 \leq t<T_{\max }}\|u(t)\|_{\mathcal{H}^{\sigma}(D)}\right\} \leq \Im .
$$

Consider a sequence of positive numbers $\left\{t_{k}\right\}_{k \in \mathbb{N}} \subset\left[0, T_{\max }\right)$ such that $t_{k} \stackrel{k \rightarrow \infty}{\longrightarrow} T_{\max }^{-}$and the sequence $\left\{u\left(t_{k}\right)\right\}$ is a subset of $\mathcal{H}^{\sigma}(D)$. We show that $\left\{u\left(t_{k}\right)\right\}_{k \in \mathbb{N}}$ is a Cauchy sequence in the Banach space $\mathcal{H}^{\sigma}(D)$. Let $t_{m}, t_{n} \in \mathcal{H}^{\sigma}(D)$, and without loss of generality suppose that $t_{m}<t_{n}$. Then, we have

$$
\begin{aligned}
u\left(t_{n}\right)-u\left(t_{m}\right)= & {\left[\mathcal{S}_{0}\left(t_{n}\right)-\mathcal{S}_{0}\left(t_{m}\right)\right] u_{0}+\int_{0}^{t_{m}}\left[\mathcal{S}\left(t_{n}-z\right)-\mathcal{S}\left(t_{m}-z\right)\right] G(u(z)) \mathrm{d} z } \\
& +\int_{t_{m}}^{t_{n}} \mathcal{S}\left(t_{n}-z\right) G(u(z)) \mathrm{d} z+\int_{t_{m}}^{t_{n}} \mathcal{S}\left(t_{n}-z\right) \int_{0}^{z} \varphi(z-s, u(s)) \mathrm{d} s \mathrm{~d} z \\
& +\int_{0}^{t_{m}}\left[\mathcal{S}\left(t_{n}-z\right)-\mathcal{S}\left(t_{m}-z\right)\right] \int_{0}^{z} \varphi(z-s, u(s)) \mathrm{d} s \mathrm{~d} z \\
= & : \mathcal{P}_{1}+\mathcal{P}_{2}+\mathcal{P}_{3}+\mathcal{P}_{4}+\mathcal{P}_{5} .
\end{aligned}
$$

Using the inequality $\left|e^{-a}-e^{-b}\right| \leq|a-b|$, we have

$$
\begin{aligned}
\left\|\mathcal{P}_{1}\right\|_{\mathcal{H}^{\sigma}(D)} & \leq \sqrt{\frac{\alpha^{2}\left(t_{n}-t_{m}\right)^{2}}{(1-\alpha)^{4}} \sum_{j=1}^{\infty} \lambda_{j}^{2 \sigma-4}\left\langle u_{0}, \psi_{j}\right\rangle_{L^{2}(D)}^{2}} \\
& \leq \frac{\alpha\left|t_{n}-t_{m}\right|}{(1-\alpha)^{2}}\left\|u_{0}\right\|_{\mathcal{H}^{\sigma-2}(D)} \leq \frac{\Im \alpha}{(1-\alpha)^{2}}\left|t_{n}-t_{m}\right| .
\end{aligned}
$$

Also

$$
\begin{aligned}
\left\|\mathcal{P}_{2}\right\|_{\mathcal{H}^{\sigma}(D)}^{2} & \leq \int_{0}^{t_{m}} \sum_{j=1}^{\infty} \frac{\lambda_{j}^{2 \sigma}\left\langle G(u(z)), \psi_{j}\right\rangle_{L^{2}(D)}^{2}}{\left(1+\lambda_{j}(1-\alpha)\right)^{4}}\left[\frac{\alpha \lambda_{j}\left(t_{n}-z\right)}{1+\lambda_{j}(1-\alpha)}-\frac{\alpha \lambda_{j}\left(t_{m}-z\right)}{1+\lambda_{j}(1-\alpha)}\right]^{2} d z \\
& \leq \frac{\alpha^{2}}{(1-\alpha)^{4}}\left(t_{n}-t_{m}\right)^{2} \int_{0}^{t_{m}}\|G(u(z))\|_{\mathcal{H}^{\nu}(D)}^{2} d z
\end{aligned}
$$




$$
\leq \frac{L_{G}^{2} \alpha^{2}\left|t_{n}-t_{m}\right|^{2}}{(1-\alpha)^{6}\left(\Im^{p}+1\right)^{-2}} T_{\max }
$$

From the same method as in the proof of Lemma 4.1 we have

$$
\left\|\mathcal{P}_{3}\right\|_{\mathcal{H}^{\sigma}(D)} \leq \frac{L_{G}}{(1-\alpha)^{2}} \int_{t_{m}}^{t_{n}}\left(\|u(z)\|_{\mathcal{H}^{\sigma}(D)}^{p}+1\right) \mathrm{d} z \leq \frac{L_{G}\left(\Im^{p}+1\right)}{(1-\alpha)^{2}}\left|t_{n}-t_{m}\right|
$$

Next, we have

$$
\begin{aligned}
\left\|\mathcal{P}_{4}\right\|_{\mathcal{H}^{\sigma}(D)} & \leq \frac{L_{\varphi}}{(1-\alpha)^{2}} \int_{t_{m}}^{t_{n}} \int_{0}^{z}\left(\|u(s)\|_{\mathcal{H}^{\sigma}(D)}^{p}+1\right) \mathrm{d} s \mathrm{~d} z \\
& \leq \frac{L_{\varphi}\left(\Im^{p}+1\right)}{(1-\alpha)^{2}} T_{\max }\left|t_{n}-t_{m}\right| .
\end{aligned}
$$

Finally note

$$
\begin{aligned}
\left\|\mathcal{P}_{5}\right\|_{\mathcal{H}^{\sigma}(D)} & \leq \frac{\alpha\left|t_{n}-t_{m}\right|}{(1-\alpha)^{2}} \sqrt{\int_{0}^{t_{m}} \int_{0}^{z}\left(\|u(z)\|_{\mathcal{H}^{\sigma}(D)}^{p}+1\right)^{2} \mathrm{~d} s \mathrm{~d} z} \\
& \leq \frac{L_{\varphi} \alpha\left|t_{n}-t_{m}\right|}{(1-\alpha)^{2}}\left(\Im^{p}+1\right) T_{\max } .
\end{aligned}
$$

From the above estimates we have

$$
\left\|u\left(t_{n}\right)-u\left(t_{m}\right)\right\|_{\mathcal{H}^{\sigma}(D)} \stackrel{m, n \rightarrow \infty}{\longrightarrow} 0 .
$$

Hence, $\left\{u\left(t_{k}\right)\right\}_{k \in \mathbb{N}}$ is a Cauchy sequence in $\mathcal{H}^{\sigma}(D)$. We deduce that $\left\{u\left(t_{k}\right)\right\}_{k \in \mathbb{N}}$ has a limit $u_{T_{\max }}$ in $\mathcal{H}^{\sigma}(D)$. Because $\left\{t_{k}\right\}_{k \in \mathbb{N}}$ is arbitrary, we have the following limit

$$
\lim _{t \rightarrow T_{\max }^{-}}\|u\|_{\mathcal{H}^{\sigma}(D)}=\left\|u_{T_{\max }}\right\|_{\mathcal{H}^{\sigma}(D)} .
$$

Therefore, we can define $u$ over $\left[0, T_{\max }\right]$. By applying Theorem 4.3, the mild solution of problem (1.1) on $\left[0, T_{\max }\right]$ can be extended to some larger interval and this leads to a contradiction with the definition of $T_{\max }$. The result follows.

\section{Numerical EXAmple}

In this section, we consider some numerical examples to illustrate two properties of the mild solution in the Fourier form (4), namely the regularity and continuation of the solution $u$ based on the time variable.

Choose $T=1$ and the domain $D=[0, \pi]$. We focus on the equation

$$
{ }_{C F} \mathscr{D}_{t}^{\alpha} u-\Delta u=G(t, x, u)+\int_{0}^{t} \varphi(t-s, u(s)) \mathrm{d} s, \quad(t, x) \in(0,1) \times(0, \pi),
$$

with the intial condition

$$
u(0, x)=u_{0}(x), x \in(0, \pi) .
$$


Then we have the orthogonal basis in $L^{2}(0, \pi)$ is $\psi_{j}(x)=\sqrt{\frac{2}{\pi}} \sin (j x)$ and the corresponding eigenvalues $\left\{\lambda_{j}\right\}=$ $\left\{j^{2} \mid j=1,2, \ldots\right\}$. The spatial variable are discretized as follows

$$
x_{l}=\frac{l \pi}{X_{l}+1}, \text { for } l=0,1, \ldots, X_{l}+1,
$$

where $X_{l}>0$ is a given integer number which is the number of partitions.

In addition, the scalar product of $g$ and $h$ in $L^{2}(0, \pi)$ is given by

$$
\langle g, h\rangle_{L^{2}(0, \pi)}=\int_{0}^{\pi} g h \mathrm{~d} x .
$$

According to (4), we have the solution as follows

$$
\begin{aligned}
& u(x, t) \\
= & \sum_{j=1}^{J}\left[\frac{1}{1+j^{2}(1-\alpha)} \exp \left(\frac{-j^{2} \alpha t}{1+j^{2}(1-\alpha)}\right) \int_{0}^{\pi} u_{0}(\xi) \psi_{j}(\xi) \mathrm{d} \xi\right. \\
& +\int_{0}^{t} \frac{\alpha}{\left[1+j^{2}(1-\alpha)\right]^{2}} \exp \left(\frac{-j^{2} \alpha(t-z)}{1+j^{2}(1-\alpha)}\right) \int_{0}^{\pi} G(z, \xi) \psi_{j}(\xi) \mathrm{d} \xi \mathrm{d} z \\
& \left.+\int_{0}^{t} \frac{\alpha}{\left[1+j^{2}(1-\alpha)\right]^{2}} \exp \left(\frac{-j^{2} \alpha(t-z)}{1+j^{2}(1-\alpha)}\right) \int_{0}^{\pi} \int_{0}^{z} \varphi(z-s, u(s, \xi)) \mathrm{d} s \psi_{j}(\xi) \mathrm{d} \xi \mathrm{d} z\right] \psi_{j}(\xi),
\end{aligned}
$$

where

$$
\begin{aligned}
& u_{0}=\sqrt{2 / \pi} \sin (x), \quad x \in[0, \pi], \\
& G(t, x)=\sqrt{2 / \pi} \sin (2 x), \quad(t, x) \in[0,1] \times[0, \pi], \\
& \varphi(w, u)=\sqrt{2 / \pi} w \sin (3 x), \quad(t, x) \in[0,1] \times[0, \pi],
\end{aligned}
$$

Next, to consider the regularity of the solution based on the time variable, we focus on the absolute error estimation between the solution at $t$ and the solution at $t^{*}$ for some values of $\alpha$ as follows

$$
A E E_{t}^{t^{*}}(\alpha)=\left(\frac{1}{X_{k}+1} \sum_{k=1}^{X_{k}+1}\left|u\left(t^{*}, x_{k}\right)-u\left(t, x_{k}\right)\right|^{2}\right)^{1 / 2}, \text { for } t \text { and } t^{*} \text { in }[0,1] \text {. }
$$

Numerical results are presented in Tables $1-4$ and Figures $1-3$, i.e., we show the solutions in the 2D and $3 \mathrm{D}$ graph for $t \in\{0.2,0.4,0.6,0.8\}, \alpha \in\{0.1,0.5,0.9\}$, respectively. From the above results, it can be concluded that the solution $u$ at $t^{*}$ approaches the solution $u$ at $t$ when $t^{*}$ tends to $t$. In addition, we also show a 3D graph of the solution $u$ for different values of $\alpha$ in $\{0.1,0.5,0.9\}$, see Figure 4 .

\section{Conclusion}

This paper considers the initial value problem for a time-fractional equation with the Caputo - Fabrizio derivative and memory effect on the source term. In our work, the formula of the mild solution is given and based on it, we investigate the global in time results when the source terms are globally Lipschitz and when the source terms are locally Lipschitz, the local in time well-posedness and finite-time blow-up results are obtained. 
TABLE 1. The error estimation between the solutions for $\alpha \in\{0.1,0.5,0.9\}$ at $t=0.2, t^{*} \in$ $\{0.21,0.205,0.201\}$.

\begin{tabular}{ccccc}
\hline \multirow{2}{*}{$t, t^{*}$} & \multicolumn{4}{c}{$X_{k}=150$} \\
\cline { 2 - 5 } & $\left|t-t^{*}\right|$ & $A E E_{t}^{t^{*}}(0.1)$ & $A E E_{t}^{t^{*}}(0.5)$ & $A E E_{t}^{t^{*}}(0.9)$ \\
\hline$\{0.2,0.21\}$ & 0.01 & 0.0012270674377 & 0.0105582080558 & 0.0636880980074 \\
$\{0.2,0.205\}$ & 0.005 & 0.0006154094608 & 0.0054654258649 & 0.0358135333112 \\
$\{0.2,0.201\}$ & 0.001 & 0.0001233859136 & 0.0011269353172 & 0.0079505003648 \\
\hline
\end{tabular}

TABLE 2. The error estimation between the solutions for $\alpha \in\{0.1,0.5,0.9\}$ at $t=0.4, t^{*} \in$ $\{0.41,0.405,0.401\}$.

\begin{tabular}{ccccc}
\hline \multirow{2}{*}{$t, t^{*}$} & \multicolumn{4}{c}{$X_{k}=150$} \\
\cline { 2 - 5 } & $\left|t-t^{*}\right|$ & $A E E_{t}^{t^{*}}(0.1)$ & $A E E_{t}^{t^{*}}(0.5)$ & $A E E_{t}^{t^{*}}(0.9)$ \\
\hline$\{0.4,0.41\}$ & 0.01 & 0.0011983825229 & 0.00855479959428 & 0.0306090673693 \\
$\{0.4,0.405\}$ & 0.005 & 0.0006009162962 & 0.00436512479262 & 0.0162930949190 \\
$\{0.4,0.401\}$ & 0.001 & 0.0001204620857 & 0.00088821424025 & 0.0034450187237 \\
\hline
\end{tabular}

TABLE 3. The error estimation between the solutions for $\alpha \in\{0.1,0.5,0.9\}$ at $t=0.6, t^{*} \in$ $\{0.61,0.605,0.601\}$.

\begin{tabular}{ccccc}
\hline \multirow{2}{*}{$t, t^{*}$} & \multicolumn{4}{c}{$X_{k}=150$} \\
\cline { 2 - 5 } & $\left|t-t^{*}\right|$ & $A E E_{t}^{t^{*}}(0.1)$ & $A E E_{t}^{t^{*}}(0.5)$ & $A E E_{t}^{t^{*}}(0.9)$ \\
\hline$\{0.6,0.61\}$ & 0.01 & 0.00117173866119 & 0.0074400470519 & 0.0208244528158 \\
$\{0.6,0.605\}$ & 0.005 & 0.00058748494398 & 0.0037778671419 & 0.0108012709801 \\
$\{0.6,0.601\}$ & 0.001 & 0.00011775757958 & 0.0007652301935 & 0.0022290448785 \\
\hline
\end{tabular}

TABLE 4. The error estimation between the solutions for $\alpha \in\{0.1,0.5,0.9\}$ at $t=0.8, t^{*} \in$ $\{0.81,0.805,0.801\}$.

\begin{tabular}{ccccc}
\hline \multirow{2}{*}{,$t^{*}$} & \multicolumn{4}{c}{$X_{k}=150$} \\
\cline { 2 - 5 } & $\left|t-t^{*}\right|$ & $A E E_{t}^{t^{*}}(0.1)$ & $A E E_{t}^{t^{*}}(0.5)$ & $A E E_{t}^{t^{*}}(0.9)$ \\
\hline$\{0.8,0.81\}$ & 0.01 & 0.00114659653167 & 0.00662422332304 & 0.0161788710335 \\
$\{0.8,0.805\}$ & 0.005 & 0.00057483220274 & 0.00335797555066 & 0.0083143933938 \\
$\{0.8,0.801\}$ & 0.001 & 0.00011521347457 & 0.00067913121516 & 0.0017010871479 \\
\hline
\end{tabular}




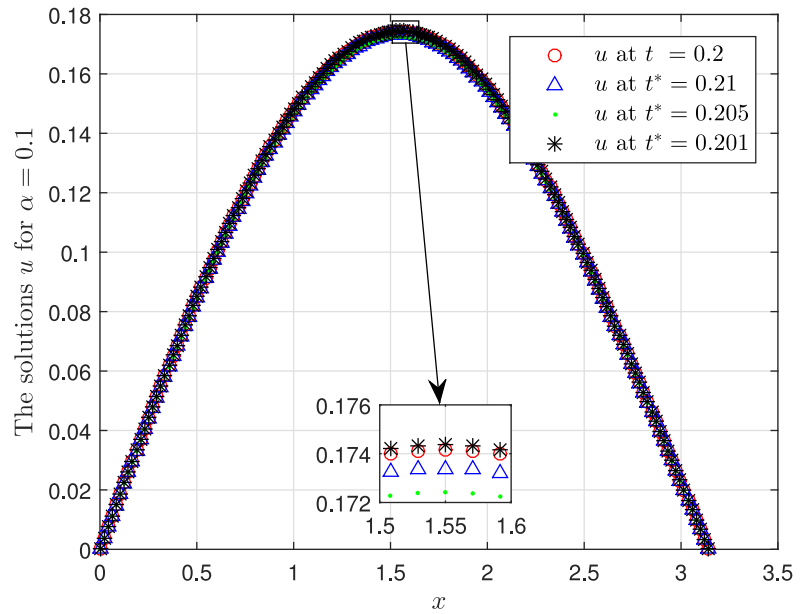

(a) The solution $u$ at $t=0.2, \alpha=0.1$

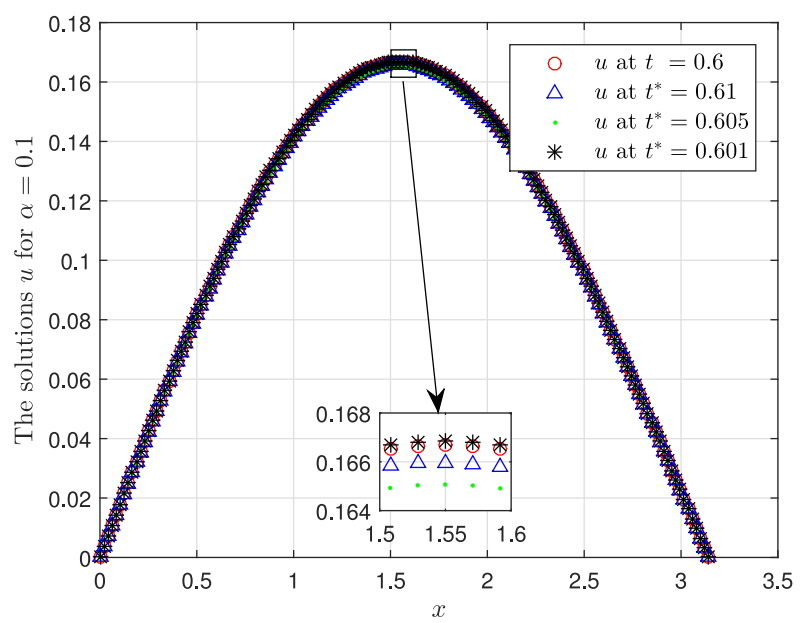

(c) The solution $u$ at $t=0.6, \alpha=0.1$

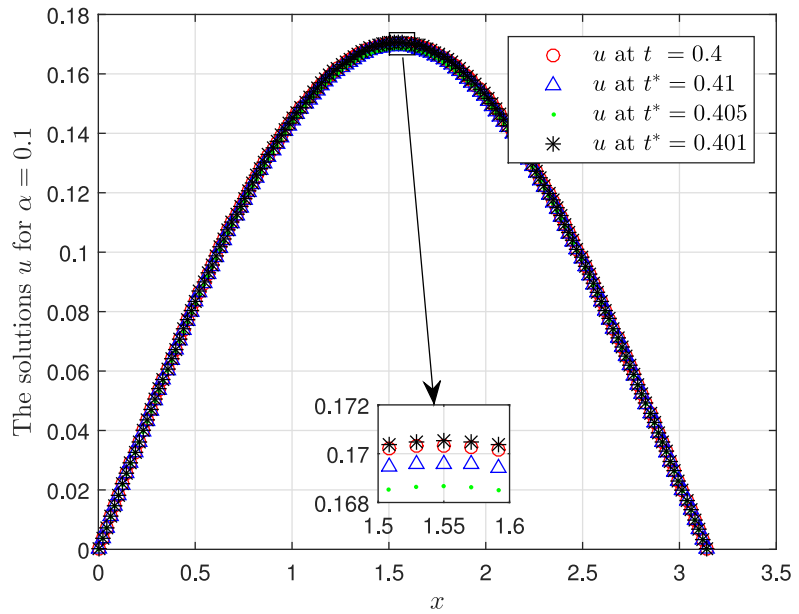

(b) The solution $u$ at $t=0.4, \alpha=0.1$

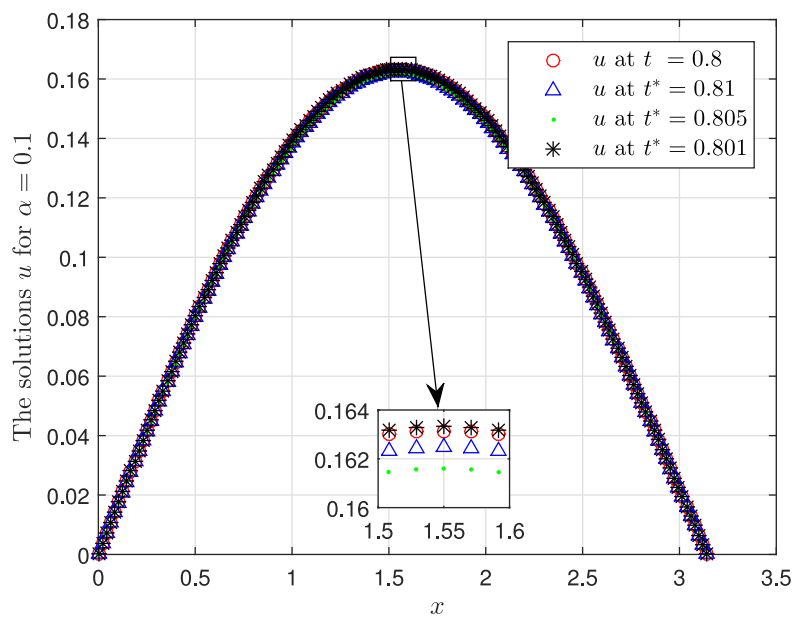

(d) The solution $u$ at $t=0.8, \alpha=0.1$

Figure 1. A comparison between the solutions $u_{\alpha}$ and $u_{\alpha^{\prime}}$ for $\alpha=0.3, \alpha^{*} \in$ $\{0.31,0.305,0.301\}$. 


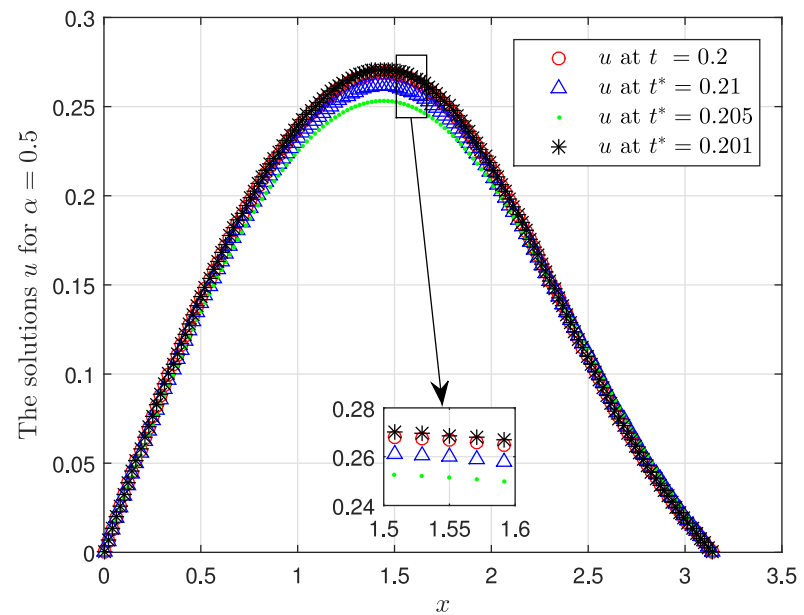

(a) The solution $u$ at $t=0.2, \alpha=0.1$

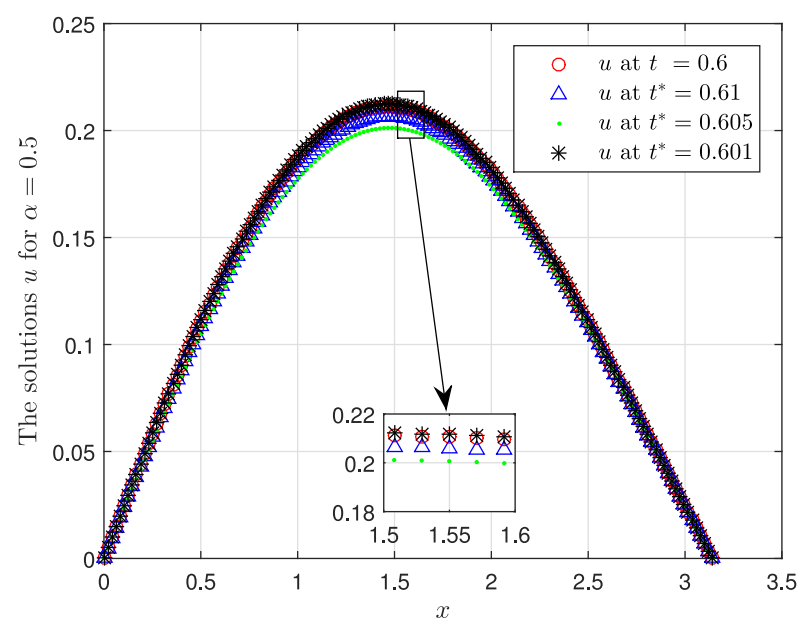

(c) The solution $u$ at $t=0.6, \alpha=0.1$

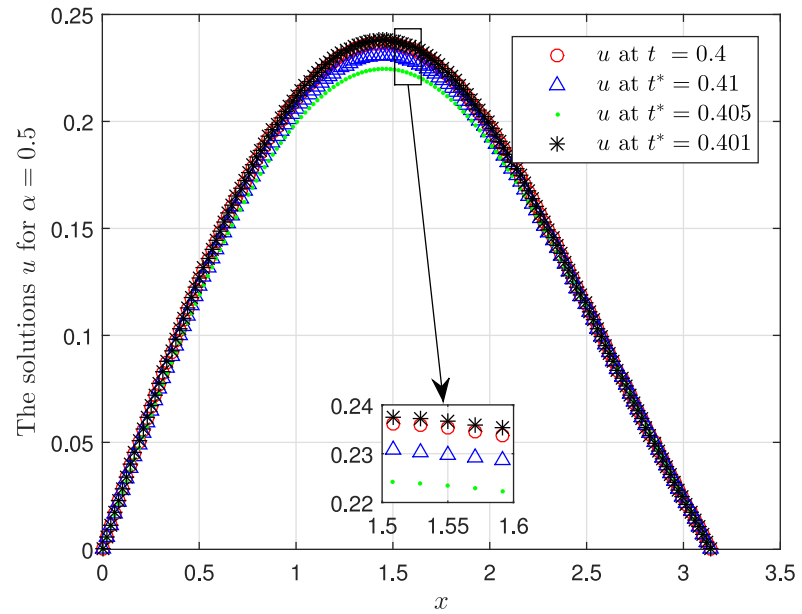

(b) The solution $u$ at $t=0.4, \alpha=0.1$

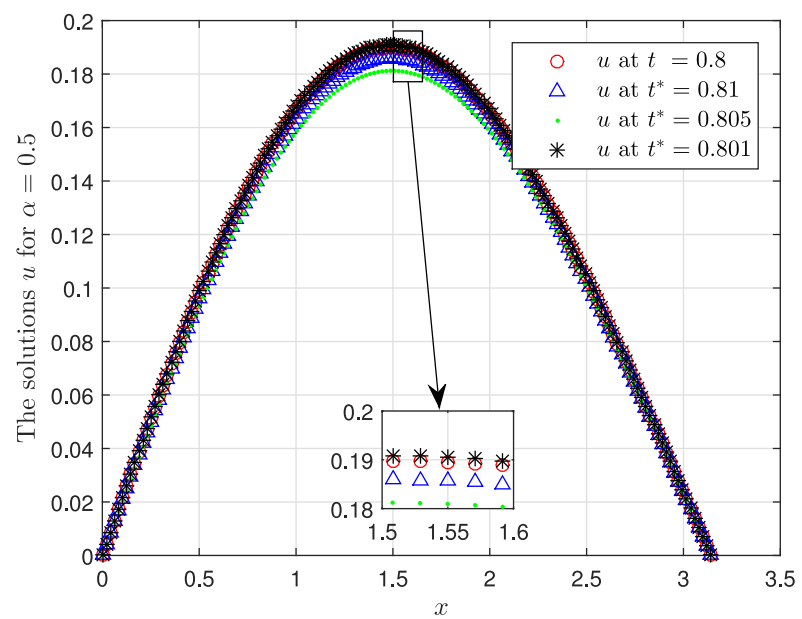

(d) The solution $u$ at $t=0.8, \alpha=0.1$

Figure 2. A comparison between the solutions $u_{\alpha}$ and $u_{\alpha^{\prime}}$ for $\alpha=0.3, \alpha^{*} \in$ $\{0.31,0.305,0.301\}$. 


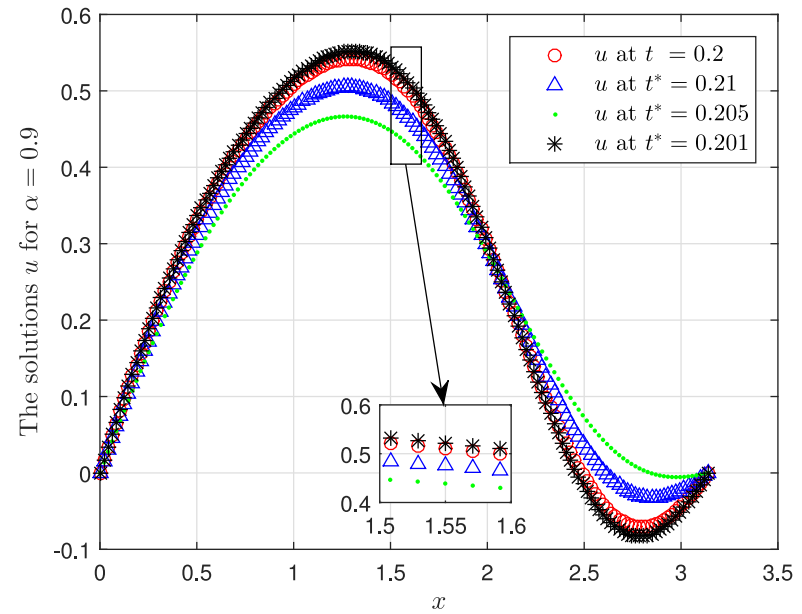

(a) The solution $u$ at $t=0.2, \alpha=0.1$

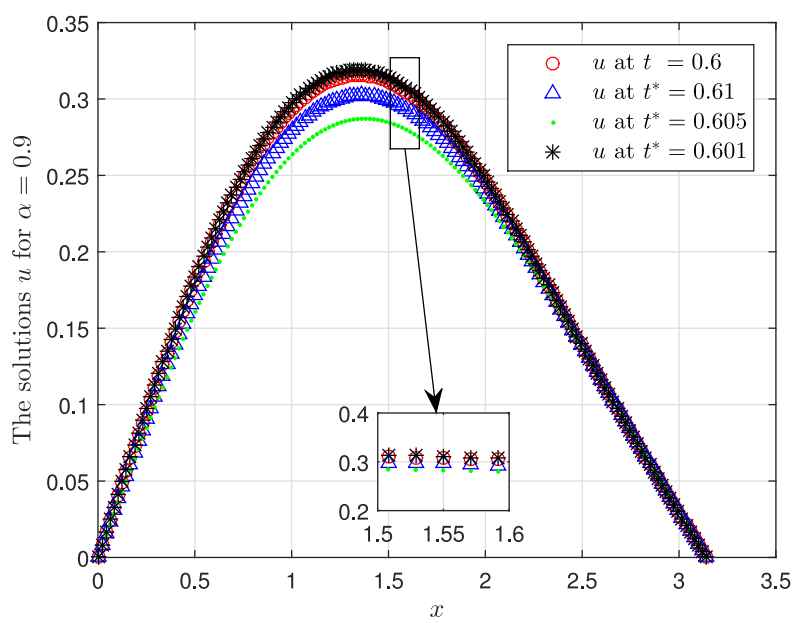

(c) The solution $u$ at $t=0.6, \alpha=0.1$

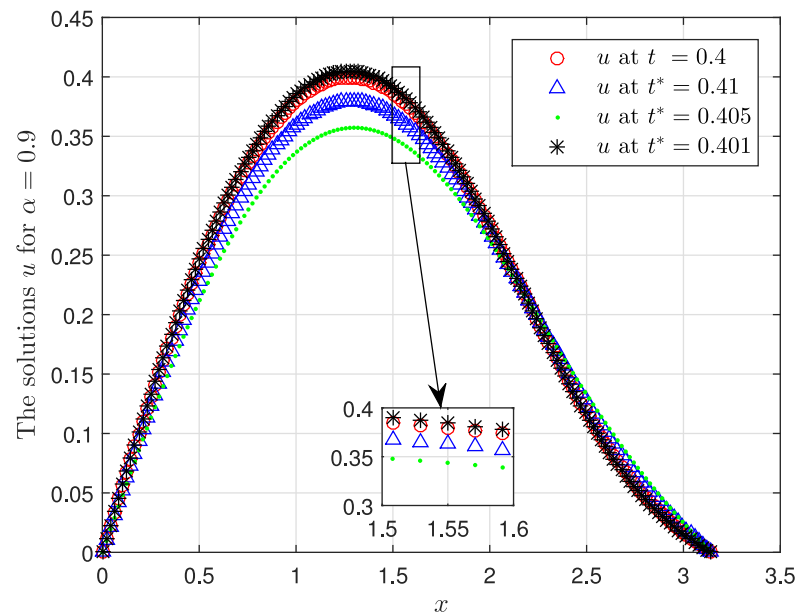

(b) The solution $u$ at $t=0.4, \alpha=0.1$

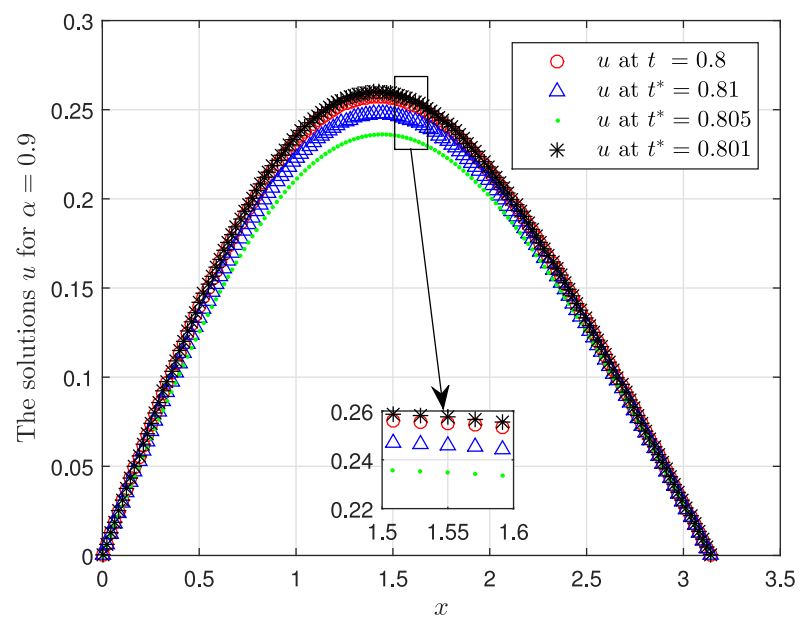

(d) The solution $u$ at $t=0.8, \alpha=0.1$

Figure 3. A comparison between the solutions $u_{\alpha}$ and $u_{\alpha^{\prime}}$ for $\alpha=0.3, \alpha^{*} \in$ $\{0.31,0.305,0.301\}$. 


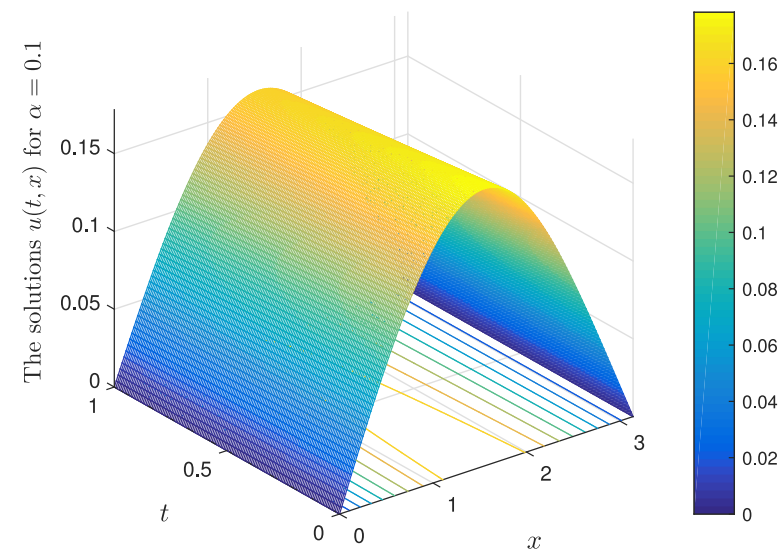

(a) 3D graph of $u$ for $\alpha=0.1$

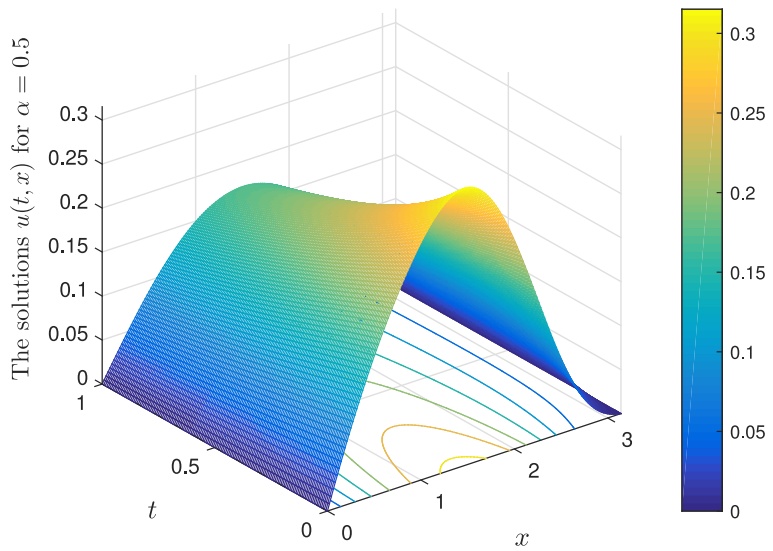

(b) 3D graph of $u$ for $\alpha=0.5$

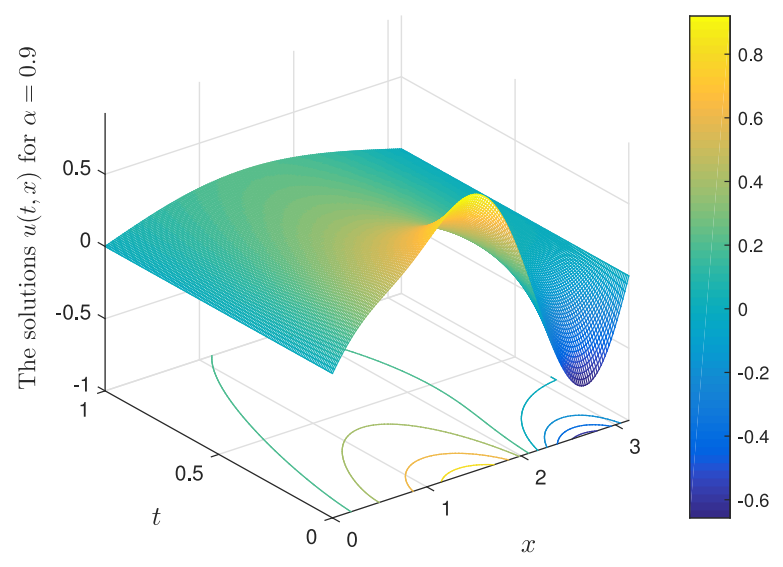

(c) 3D graph of $u$ for $\alpha=0.9$

Figure 4. The 3D graph of the solutions $u$ on $(t, x) \in(0,1) \times(0, \pi)$ for $\alpha \in\{0.1,0.5,0.9\}$.

\section{REFERENCES}

[1] K. Aissania, M. Benchohrab and J.J. Nieto, Controllability for impulsive fractional evolution inclusions with state-dependent delay. Adv. Theory Nonlinear Anal. Appl. 3 (2019) 18-34.

[2] N. Al-Salti, E. Karimov and K. Sadarangani, On a differential equation with Caputo-Fabrizio fractional derivative of order $1<\beta \leq 2$ and application to mass-spring-damper system. Progr. Fract. Differ. Appl. 2 (2017) 257-263.

[3] B. Andrade and A. Viana, Abstract Volterra integrodifferential equations with applications to parabolic models with memory. Math. Ann. 369 (2017) 1131-1175.

[4] B. Andrade and A. Viana, Integrodifferential equations with applications to a plate equation with memory. Math. Nachr. 289 (2016) 2159-2172.

[5] D. Baleanu, S. Etemad and S. Rezapour, A hybrid Caputo fractional modeling for thermostat with hybrid boundary value conditions. Bound. Value Probl. 2020 (2020) 64.

[6] D. Baleanu, S. Etemad, S. Pourrazi and S. Rezapour, On the new fractional hybrid boundary value problems with three-point integral hybrid conditions. Adv. Differ. Equ. 2019 (2019) 473.

[7] D. Baleanu, H. Mohammadi and S. Rezapour, Analysis of the model of HIV-1 infection of $C D 4+$ T-cell with a new approach of fractional derivative. Adv Differ Equ. 2020 (2020) 71.

[8] D. Baleanu, A. Mousalou and S. Rezapour, The extended fractional Caputo-Fabrizio derivative of order $0 \leq \sigma<1$ on $C_{\mathbb{R}}[0,1]$ and the existence of solutions for two higher-order series-type differential equations. Adv. Differ. Equ. 2018 (2018) 255. 
[9] D. Baleanu, A. Jajarmi, H. Mohammadi and S. Rezapour, A new study on the mathematical modelling of human liver with Caputo-Fabrizio fractional derivative. Chaos, Solitons Fractals 134 (2020) 109705.

[10] D. Baleanu, A Jajarmi, S.S. Sajjadi and J.H. Asad, The fractional features of a harmonic oscillator with position-dependent mass. Commun. Theor. Phys. 72 (2020) 055002.

[11] D. Baleanu, S. Rezapour and Z. Saberpour, On fractional integro-differential inclusions via the extended fractional CaputoFabrizio derivation. Bound. Value Probl. 2019 (2019) 79.

[12] D. Baleanu, S. Rezapour and H. Mohammadi, Some existence results on nonlinear fractional differential equations. Philos. Trans. R. Soc. Lond. Ser. A Math. Phys. Eng. Sci. 371 (2013) 20120144.

[13] E.G. Bazhlekova, Subordination principle for fractional evolution equations. Fract. Calc. Appl. Anal. 3 (2000) $213-230$.

[14] Y. Cao, J. Yin and C. Wang, Cauchy problems of semilinear pseudo-parabolic equations. J. Differ. Equ. 246 (2009) 4568-4590.

[15] M. Caputo and M. Fabrizio, applications of new time and spatial fractional derivatives with exponential kernels. Progr. Fract. Differ. Appl. 2 (2016) 1-11.

[16] M. Caputo and M. Fabrizzio, A new definition of fractional derivative without singular Kernel. Prog. Fract. Differ. Appl. 1 (2015) 73-85.

[17] T. Caraballo and J. Real, Attractors for 2D-Navier-Stokes models with delays. J. Differ. Equ.. 205 (2004) $271-297$.

[18] T. Caraballo and J. Real, Asymptotic behaviour of two-dimensional Navier-Stokes equations with delays. R. Soc. Lond. Proc. Ser. A Math. Phys. Eng. Sci. 459 (2003) 3181-3194.

[19] Y. Chen, H. Gao, M. Garrido-Atienza and B. Schmalfuß, Pathwise solutions of SPDEs driven by Hölder-continuous integrators with exponent larger than $1 / 2$ and random dynamical systems. Discr. Continu. Dyn. Syst. Series A. 34 (2014) $79-98$.

[20] M. Conti, E. Marchini and V. Pata, Reaction-diffusion with memory in the minimal state framework. Trans. Amer. Math. Soc. 366 (2014) 4969-4986.

[21] M. Conti, E. Marchini and V. Pata, A well posedness result for nonlinear viscoelastic equations with memory. Nonlinear Anal. 94 (2014) 206-216.

[22] M. D'Abbicco, The influence of a nonlinear memory on the damped wave equation. Nonlinear Anal. 95 (2014) $130-145$.

[23] J.E.L. Delgado, J.E.S. Perez, J.F.G. Aguilar and R.F.E. Jiménez, A new fractional-order mask for image edge detection based on Caputo-Fabrizio fractional-order derivative without singular kernel. Circuits Syst. Signal Process 39 (2020) $1419-1448$.

[24] M. Fabrizio and S. Polidoro, asymptotic decay for some differential systems with fading memory. Appl. Anal. 81 (2002) $1245-1264$.

[25] E. Franc and D. Goufo, Application of the Caputo-Fabrizio fractional derivative without singular kernel to Korteweg-de Vries-Burgers equation. Math. Model. Anal. 21 (2016) 188-198.

[26] A. Jajarmi and D. Baleanu, A new iterative method for the numerical solution of high-order non-linear fractional boundary value problems. Front. Phys. 8 (2020) 220.

[27] A. Jajarmi and D. Baleanu, On the fractional optimal control problems with a general derivative operator. Asian J. Control. 23 (2021) 1062-1071.

[28] T.H. Kaddoura and A. Hakema, Sufficient conditions of non global solution for fractional damped wave equations with non-linear memory. Adv. Theory Nonlinear Anal. Appl. 2 (2018) 224-237.

[29] H. Khan, J.F.G. Aguilar, T. Abdeljawad and A. Khan, Existence results and stability criteria for ABC-fuzzy-Volterra integrodifferential equation. Fractals 28 (2020) 9 pp.

[30] A. Lunardi, on the linear heat equation with fading memory. SIAM J. Math. Anal. 21 (1990) 1213-1224.

[31] E. Meerschaert, E. Nane and P. Vellaisamy, Fractional Cauchy problems on bounded domains. Ann. Probab. 37 (2009) 9791007.

[32] F. Mohammadi, L. Moradi, D. Baleanu and A Jajarmi, A hybrid functions numerical scheme for fractional optimal control problems: application to non-analytic dynamical systems. J. Vib. Control 24 (2018) 5030-5043.

[33] D. Mozyrska, D.F.M. Torres and M. Wyrwas, Solutions of systems with the Caputo-Fabrizio fractional delta derivative on time scales. Nonlinear Anal. Hybrid Syst. 32 (2019) 168-176.

[34] J.E. Muñoz Rivera and L.H. Fatori, Smoothing effect and propagations of singularities for viscoelastic plates. J. Math. Anal. Appl. 206 (1997) 397-427.

[35] A. Nabti, Life span of blowing-up solutions to the Cauchy problem for a time-space fractional diffusion equation. Comput. Math. Appl. 78 (2019) 1302-1316.

[36] S. Sajjadi, D. Baleanu, A. Jajarmi and H.M. Pirouz, A new adaptive synchronization and hyperchaos control of a biological snap oscillator. Chaos Solitons Fractals. 138 (2020) 109919.

[37] S. Samko and J.J. Trujillo, On the existence of blow up solutions for a class of fractional differential equations. Fract. Calc. Appl. Anal. 18 (2015) 281-283.

[38] N.H. Tuan, M. Hakimeh and S. Rezapour, A mathematical model for COVID-19 transmission by using the Caputo fractional derivative. Chaos Solitons Fractals 140 (2020) 110107.

[39] N.H. Tuan and Z. Yong, Well-posedness of an initial value problem for fractional diffusion equation with Caputo-Fabrizio derivative. J. Comput. Appl. Math. 375 (2020) 112811.

[40] A. Viana, Local well-posedness for a Lotka-Volterra system in Besov spaces. Comput. Math. Appl. 69 (2015) 667-674. 